\section{Traut Euch!}

\section{Liebe Leserinnen und Leser,}

Controller tun sich mit dem Personalbereich traditionell schwer. $\mathrm{Zu}$ verschieden sind die jeweiligen Denkmuster und Herangehensweisen, zu groß die gegenseitigen Vorurteile. Dabei sind sich die beiden Bereiche doch eigentlich ganz ähnlich. Beide haben zunehmend den Anspruch, das Management als Business Partner auf Augenhöhe zu unterstützen. Gleichzeitig werden beide von außen gerne veräppelt („Personalfutzis“, „Erbsenzähler“) und müssen zunehmend nicht nur den mit ihnen verbundenen Gemeinkostenblock rechtfertigen, sondern auch die Grenzen von Automatisierung und Shared Service ausloten.

Vor allem mit Blick auf die Kooperation beider Bereiche wundert man sich als unbefangener Beobachter. Erwarten würde man, dass Personaler und Controller eng zusammenarbeiten. Personalthemen sind nicht nur ein zentraler Kostenblock, sondern auch ein wichtiger Treiber einer effektiven Strategieumsetzung und damit integraler Bestandteil der Unternehmenssteuerung. Zudem wissen wir aus unseren empirischen Studien, dass eine enge Zusammenarbeit verschiedener Funktionen im Unternehmen ein wesentlicher Erfolgstreiber ist. Faktisch beschränkt sich der Austausch zwischen Personalabteilung und Controlling aber in der Regel auf wenige zentrale Größen wie Personalkosten und Vollzeitarbeitsplätze. Die Planung von Größen wie Diversity oder Mitarbeiterzufriedenheit erfolgt hingegen zumeist autonom im Personalbereich. Wer das Konzept der Balanced Scorecared mit seiner Verknüpfung von finanziellen und nicht-finanziellen strategischen Zielen kennt, muss sich spätestens an dieser Stelle wundern. Würde man nicht erwarten, dass personalbasierte Erfolgsfaktoren in die Unternehmenssteuerung integriert werden und die beiden Bereiche dabei eng zusammenarbeiten? Weit gefehlt! In der Regel beschränken sich Controller auf das Kostenthema. Würde man nicht weiter erwarten, dass das Controlling den strategischen Erfolgsbeitrag „moderner“ Konzepte wie Diversity quantitativ unterlegt oder gegebenenfalls infrage stellt? Wieder weit gefehlt. In der Regel will das der Vorstand, und das reicht.

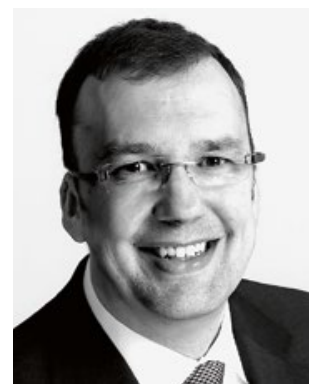

Utz Schäffer

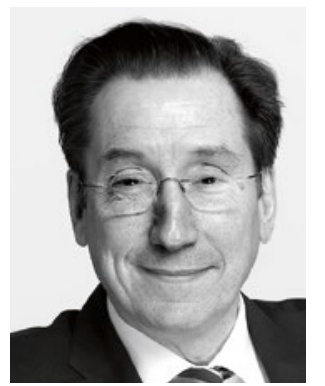

Jürgen Weber

Aus der Perspektive der meisten Controller ist der Fall klar: Es gibt viel - zu viel - zu tun, und niemand fordert ein Mehr an Zusammenarbeit mit dem Personalbereich entschieden ein. Warum also die Mühe? Ist gut nicht gut genug? Die Entscheidung liegt natürlich ganz bei Ihnen. Das Potenzial für ein Mehr an Zusammenarbeit ist aber fraglos da, und es ist nicht auf Steuerungsfragen beschränkt. Weitere Themen, die förmlich nach mehr Kooperation schreien, sind Restrukturierungsprojekte und die anstehende Digitalisierung ganzer Geschäftsprozesse. Hier können Spezialkenntnisse der Fachabteilung und die Controller-Expertise im Handling von Daten und analytischen Methoden genauso produktiv zusammenspielen wie beim vergleichsweise jungen Thema Business Analytics. Vielleicht wollen Sie es ja gemeinsam mit Ihrem Personaler anpacken.

Viel Spaß bei der Lektüre wünschen Ihnen

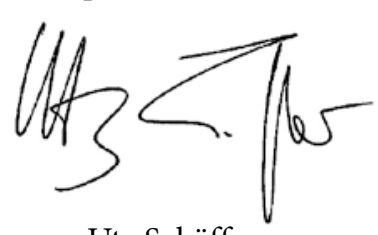

Utz Schäffer

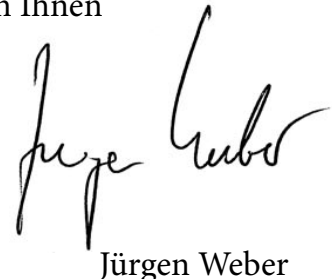

Jürgen Weber 